\title{
OBSTACLE-AVOIDING PATH PLANNING FOR HIGH VELOCITY WHEELED MOBILE ROBOTS
}

\author{
Jorge Villagra* Hugues Mounier ${ }^{* *}$ \\ * PSA-Peugeot-Citroën, \\ DINQ/DRIA/SARA/EMSA/EEES \\ Route de Gisy, 78943 Vélizy Villacoublay Cedex, FRANCE \\ ** Institut d'Electronique Fondamentale, Bâtiment 220 \\ Université Paris-Sud, 91405 Orsay, FRANCE
}

\begin{abstract}
This paper presents a new motion planning algorithm for wheeled mobile robots in presence of known static obstacles, especially well-suited for high velocity situations. It takes into account several conditions traditionally attached to smooth path planning, i.e. paths with continuous derivative and upper-bounded curvature. It makes use of a global path planner which exploits polynomial $G^{3}$ curves characteristics. Copyright (C)2005 IFAC
\end{abstract}

Keywords: vehicle dynamics, path planning, splines, smoothness criterion, obstacle avoidance

\section{INTRODUCTION}

Path planners for wheeled mobile robots (i.e vehicles that ensure non-holonomic kinematic constraints) have been widely developed in the recent years.

Dubins' pioneer work (Dubins, 1957) introduced the first set of paths going from a point to another in a minimal time (circles and segments). In this scope (path planning in a free environment), several extensions of this result have been carried out. Firstly (Reeds and Shepp, 1990) generalized Dubins' result for a forward/backward mobile. Numerous approaches have been proposed since then, based on line segments and circles, e.g. (Jacobs and Canny, 1989), (Simeon et al., 1997), (Mirtich, 1992).

All these results turned out to be unsatisfactory when implemented on real wheeled robots, because of discontinuities on path's curvature profile during circle-segment transitions. This means that the steering wheel angle has to change in- stantaneously whenever a joint is passed, which is neither physically possible nor desirable. Later (Sussmann, 1997) solved a modified version of the Dubins's Markov problem, in which the control is angular acceleration rather than angular velocity. When using the Pontryaguin Maximum Principle to solve this problem, a first analysis suggests that the manoeuvres resulting from the solution are bang-bang trajectories (as it happens in the simple Dubins' Problem), and it is reasonable to guess that these trajectories will have a finite number of switches. However, it can be proved that optimal trajectories for this kind of system involve infinite chattering. In order to avoid this problem, several works have been developed. They all try to obtain smoother trajectories than those supplied by Dubins's curves, while guaranteeing "nearly timeoptimal paths". For example, (Anisi, 2003) suggests that for generating such nearly time-optimal paths, an appropriate and cunning choice of the integral cost function has to be done. For his part, (Scheuer, 1998) uses the set of optimal curves described in Sussman's paper (segments, arcs of 
circles and clothoids); he implemented an algorithm based on Dubins' curves families, modifying single turns by continuous-curvature (CC) turns (with the help of those clothoids). However such a path cause discontinuities in the steering wheel angular velocity of a vehicle covering the path. This is why many researches have been carried out in order to find curves with a higher degree of smoothness. For instance, (Kanayama and Hartman, 1989) showed that using cost functions like the integral over the curvature's square or the integral over the square of curvature derivative, lead, respectively, concatenations of clothoids, and concatenation of cubic spirals. Many other sets of fundamentals curves have been tested:

- Some whose coordinates have a closed expression: B-splines (Komoriya and K.Tanie, 1989), quintic polynomials (Takahashi et al., 1989), polar splines (Nelson, 1989).

- Others whose curvature is a function of their arc length: clothoids (Liscano and Green, 1989), Kanayama \& Hartmann curves, intrinsic splines (Delingette et al., 1991) or $G^{2}$ splines (Piazzi et al., 2002).

As neither completeness nor topological admissibility have been studied in depth, none seemed to be more interesting than others. Furthermore, works combining smooth path planning and obstacle-avoiding have so far barely been developed. Only ${ }^{1}$ (Berglund et al., 2003) and (Scheuer, 1998) have treated this problem, the first one using an heuristic choice on the curve family (B-splines), the second one supported by Sussmann's results, the latter is closest to optimality.

The main drawback of Scheuer's result is that his path's curvature profile is too constrained for difficult situations, and that every circle-segment transition generated by clothoids is not smooth enough (curvature is not continuously differentiable).

Consequently, a more versatile local path planner will be proposed (based on results from (Piazzi et al., 2003)), to use in a similar way as in Scheuer's strategy, i.e., embedded in a probabilistic path planner. It will provide near optimal paths with smooth curvature profiles and with bounded curvature and curvature derivative.

\subsection{Outline of the paper}

Section 2 will present the general path planning problem, introducing the two fundamental

\footnotetext{
1 Laumond's publication (Laumond et al., 1994) on Hilare robot also deals with this problem, but for his case maximal curvature constraint is not taken into account because the robot has an unbounded turning radius
}

algorithms developed for its resolution. First, in section 3 , the $G^{3}$ quintic splines curves are defined, highlighting their applicability to a smooth path planning algorithm (in a free environment). Obstacle-avoiding is treated in section 4, where the global path planner developed is described. The final section is dedicated to a comparison between $G^{3}$ splines and CC curves and, finally, to show a path generation application over an environment with obstacles.

\section{THE PATH PLANNING PROBLEM}

Given a starting and a goal configuration, the path planning problem consists in finding a path $\Gamma$ such that:

(1) $\Gamma$ satisfies the extreme conditions (explained in detail in the local path planner section)

(2) $\Gamma$ respects local path planner constraints, i.e kinematic and dynamic vehicle constraints.

(3) $\Gamma$ is collision-free, i.e. it is entirely included in the free configuration space.

Among the set of possible solutions, the shortest path will be chosen. Moreover, as it will be explained at the end of section 4 , if there exists a solution to the above formulated problem, the algorithm will find it.

\section{LOCAL PATH PLANNER}

A curve on the $\{x, y\}$-plane can be parameterized by means of $\mathbf{p}:\left[u_{0}, u_{1}\right] \rightarrow \mathbb{R}^{2}, u \rightarrow \mathbf{p}(u)=$ $[x(u), y(u)]^{T}$. The curve length measured along $\mathbf{p}(u)$, denoted by $s$ can be expressed as

$$
\begin{aligned}
& f:\left[u_{0}, u_{1}\right] \rightarrow[0, f(u)], \\
& u \mapsto s=\int_{u_{0}}^{u}\|\dot{\mathbf{p}}(\xi)\| d \xi=\int_{u_{0}}^{u} \sqrt{\dot{x}(\xi)^{2}+\dot{y}(\xi)^{2}} d \xi
\end{aligned}
$$

Given a regular curve ${ }^{2}\left(\dot{\mathbf{p}}(u) \in C_{p}\left(\left[u_{0}, u_{1}\right]\right)\right.$, $\left.\dot{\mathbf{p}}(u) \neq 0, \forall u \in\left[u_{0}, u_{1}\right]\right)$, the length function $f$ is continuous over $\left[u_{0}, u_{1}\right]$ and bijective, hence its inverse is continuous too and will be represented by $f^{-1}:\left[0, f\left(u_{1}\right)\right] \rightarrow\left[u_{0}, u_{1}\right], s \rightarrow u=f^{-1}(s)$.

An orthonormal moving frame $\{\tau(u), \nu(u)\}$ congruent with the axes of plane may be associated with every point of the curve. The unit tangent vector of the curve is defined as $\tau=\frac{\dot{u}}{\|\dot{u}\|}$, and its derivative with respect to the curve length, according to Frenet formulae, can be written as $\frac{d \tau}{d s}(u)=\kappa_{c}(u) \nu(u), u \in\left[u_{0}, u_{1}\right]$. The scalar curvature $\left(\kappa_{c}\right)$ may be expressed either as a function of $u$ :

$$
k(u)=\frac{\dot{x}(u) \ddot{y}(u)-\ddot{x}(u) \dot{y}(u)}{\left(\dot{x}(u)^{2}+\dot{y}(u)^{2}\right)^{3 / 2}},
$$

\footnotetext{
$2 C_{p}$ is the class of piecewise continuous functions.
} 
or as a function of the curve length, i.e. $\kappa$ : $\left[0, f\left(u_{1}\right)\right] \rightarrow \mathbb{R}, \quad u \mapsto \kappa(s)$ where $\kappa(s)=$ $\kappa_{c}\left(f^{-1}(s)\right)$.

Definition 1. A parametric curve $\mathbf{p}(\mathrm{u})$ has first order geometric continuity $\left(G^{1}\right)$ if it verifies: $\dot{\mathbf{p}}(u) \in C_{p}\left(\left[u_{0}, u_{1}\right]\right), \dot{\mathbf{p}}(u) \neq 0, \forall u \in\left[u_{0}, u_{1}\right]$ and $\tau(\cdot) \in C^{0}\left(\left[u_{0}, u_{1}\right]\right)$.

Definition 2. A parametric curve $\mathbf{p}(\mathrm{u})$ has second order geometric continuity $\left(G^{2}\right)$ if it is a $G^{1}$ curve, $\ddot{\mathbf{p}}(u) \in C_{p}\left(\left[u_{0}, u_{1}\right]\right)$ and $\kappa_{c}(\cdot) \in C^{0}\left(\left[u_{0}, u_{1}\right]\right)$.

Definition 3. A parametric curve $\mathbf{p}(\mathrm{u})$ has third order geometric continuity $\left(G^{3}\right)$ if it is a $G^{2}$ curve, $\dddot{\mathbf{p}}(u) \in C_{p}\left(\left[u_{0}, u_{1}\right]\right)$ and $\ddot{\tau}_{c}(\cdot) \in C^{0}\left(\left[u_{0}, u_{1}\right]\right)$.

Definition 4. A path $\Gamma$ on the $\{x, y\}$ plane is a $G^{i}$-path $(\mathrm{i}=1,2,3)$ if there exists a parametric $G^{i}$ curve whose image $\Gamma$ is the given path

Proposition 1. A path $\Gamma$ on the $\{x, y\}$ plane is generated by the unicycle model:

$$
\left\{\begin{array}{l}
\dot{x}=v(t) \cos \theta(t) \\
\dot{y}=v(t) \sin \theta(t) \\
\dot{\theta}=\omega(t)
\end{array}\right.
$$

with input $\omega(\cdot) \in \mathcal{C}^{1}, v(t)=V_{0}, V_{0} \in \mathbb{R}^{+}, \forall t \geq$ 0 , if and only if it is a $G^{3}$ curve.

A sketch of the proof is as follows. Given any $G^{3}$-curve $\mathbf{p}(u)$ with $u \in\left[u_{0}, u_{1}\right]$, the inverse arc length function $s^{-1}$ is defined, and it is, by definition, a continuous function. Moreover, the scalar curvature derivative $\sigma(u)=\frac{d \kappa}{d u}(u)$ is as well continuous over $\left[u_{0}, u_{1}\right]$ because $\mathbf{p}(u)$ is a $G^{3}$ curve. At the initial time $t_{0}$, consider the state of model (3) given by $\left[x\left(u_{0}\right) y\left(u_{0}\right) \arg \left(\tau\left(u_{0}\right)\right)\right]^{T}$. Then, applying the continuous input

$$
\omega(t)=v \kappa_{c}\left(s^{-1}\left(v\left(t-t_{0}\right)\right)\right)
$$

the vehicles motion from $t_{0}$ to $t_{0}+\frac{s\left(u_{1}\right)}{v}$ exactly matches the path of the given curve. Moreover, if $\kappa_{c}(u) \in C^{1}$ and $\dot{v}=0$, then $\omega(u) \in C^{1}$.

As it has been said in the introduction, several possibilities could have been used in order to guarantee $G^{3}$ curve properties (B-splines are $G^{3}$ curves; CC curves only guarantee $G^{2}$ properties), while approaching time-optimality. Let's for instance consider a quintic polynomial curve $\mathbf{p}(u)=[x(u), y(u)]^{T}, u \in[0,1]$, where

$$
\begin{aligned}
& x(u)=x_{0}+x_{1} u+x_{2} u^{2}+x_{3} u^{3}+x_{4} u^{4}+x_{5} u^{5} \\
& y(u)=y_{0}+y_{1} u+y_{2} u^{2}+y_{3} u^{3}+y_{4} u^{4}+y_{5} u^{5}
\end{aligned}
$$

The problem is then to determine the minimalorder (and not superior to the fifth order) polynomial curve that interpolates between two points
$\mathbf{p}_{A}=\left[x_{A}, y_{A}\right]^{T}$ and $\mathbf{p}_{B}=\left[x_{B}, y_{B}\right]^{T}$, with angles $\theta_{A}$ and $\theta_{B}$ defining its tangent vector, with scalar curvatures $\kappa_{A}$ and $\kappa_{B}$, and with derivatives of the curvature with respect to the arc length $\dot{\theta}_{A}$ and $\dot{\theta}_{B}$. It follows that, for every two points $A$ and $B$, the interpolating conditions are the following:

$$
\begin{aligned}
& \mathbf{p}(0)=p_{A}, \quad \tau(0)=\left[\begin{array}{c}
\cos \theta_{A} \\
\sin \theta_{A}
\end{array}\right], \quad \kappa_{c}(0)=\kappa_{A}, \\
& \sigma_{c}(0)=\sigma_{A}\|\dot{\mathbf{p}}(0)\| \\
& \mathbf{p}(1)=p_{B}, \quad \tau(1)=\left[\begin{array}{c}
\cos \theta_{B} \\
\sin \theta_{B}
\end{array}\right], \quad \kappa_{c}(1)=\kappa_{B}, \\
& \sigma_{c}(1)=\sigma_{B}\|\dot{\mathbf{p}}(1)\|
\end{aligned}
$$

A non degenerate nonlinear system of 12 equations can be written from the interpolating conditions. As the number of coefficients to find is also 12 , a determined solution can be found.

Furthermore, a two dimensional vector $\eta \equiv$ $\left[\eta_{1}, \eta_{2}\right]^{T}$ is defined with respect to the polynomial coefficients $\left(x_{i}, y_{i}\right)$. Both parameters $\eta_{1}$ and $\eta_{2}$ allow to reparametrize the curve $\mathbf{p}(u)$ as what is called an $\eta$-spline, denoted $\mathbf{p}(u, \eta)$. They are defined as follows:

$$
\eta_{1}:=\|\dot{\mathbf{p}}(0)\|, \quad \eta_{2}:=\|\dot{\mathbf{p}}(1)\|
$$

so they may be considered as the velocity parameters at the beginning and end of the curve.

Proposition 2. Given any two points and its corresponding data $\mathbf{p}_{A}, \theta_{A}, \kappa_{A}, \dot{\kappa}_{A}$ and $\mathbf{p}_{B}, \theta_{B}, \kappa_{B}$, $\dot{\kappa}_{B}$, the parametric curve $\mathbf{p}(u, \eta)$ satisfies interpolation conditions above (5)-(6) for all $\eta \in \mathcal{H}$. On the contrary, for any fifth order polynomial curve $\mathbf{p}(u)$ with $\dot{\mathbf{p}}(0) \neq 0, \dot{\mathbf{p}}(1) \neq 0$ satisfying (5)-(6), there exists a parameter vector $\eta \in \mathcal{H}$ such that the curve $\mathbf{p}(u)$ can be expressed as $\mathbf{p}(u, \eta)$.

The choice of both $\eta_{1}$ and $\eta_{2}$ is then the last point to clear up.

It is quite intuitive that with a proper selection of $\eta$ it is possible to obtain a large variety of curves, all of them interpolating the conditions imposed at the curve extremes. This suggests to choose both parameters with some sort of optimality criterion. In their work, (Bianco and Piazzi, 2000) pointed out the advantage of minimizing $\left\|\frac{d \kappa}{d s}\right\|$, based on the system flatness properties. According to them, the optimization problem can be formulated as follows

$$
\min _{\eta \in \mathcal{H}} \max _{s \in\left[0, f^{-1}(u)\right]}\left\|\frac{d k}{d s}(s)\right\|,
$$

where $\mathcal{H}$ is the set given by the Cartesian product $\mathbb{R}^{+} \times \mathbb{R}^{+}$. This problem formulation, considering (1), is equivalent to this other constrained minmax problem: 


$$
\begin{aligned}
& \min _{\eta \in \mathcal{H}} \max _{u \in[0,1]}\left\|\frac{d k}{d s}(u)\right\| \\
& \|\dot{\mathbf{p}}(u)\|>0 \forall u \in[0,1]
\end{aligned}
$$

The purpose of such criteria is to minimize, under reasonable approximations, the absolute value of the derivative of the curvature with respect to time. The underlying idea of this choice is to limit as much as possible the steering speed. Even though this argument is admissible from a theoretic point of view, it is not constraining enough if a minimum turning radius have to be fulfilled (which is a technological imperative). This is why this other problem formulation is preferable:

$$
\begin{aligned}
& \min _{\eta \in \mathcal{H}} \max _{u \in[0,1]}\left\|\frac{d k}{d s}(u)\right\| \\
& \|\dot{\mathbf{p}}(u)\|>0 \\
& \|\kappa(u)\| \leq \kappa_{\max }
\end{aligned}
$$

Since the derivative of the curvature is related to the steering speed, it is desirable to upperbound the first one in order to guarantee such paths to be tracked within a given speed. In other words, the covered trajectory by the unicycle model becomes more realistic (when compared with a real vehicle) if a constraint $\left|\frac{\mathrm{d} \kappa}{d s}(s)\right| \leq$ $\sigma_{\max }$ is imposed to the reference path. Thus, as we look for the minimal length trackable path, the previous problem formulation turn into the following:

$$
\begin{aligned}
& \min _{\eta \in \mathcal{H}} \int_{u_{0}}^{u} \sqrt{\dot{x}(\xi)^{2}+\dot{y}(\xi)^{2}} d \xi \\
& \|\dot{\mathbf{p}}(u)\|>0 \\
& \|\kappa(u)\| \leq \kappa_{\max } \\
& \left\|\frac{d \kappa}{d u}(u)\right\| \leq \sigma_{\max }
\end{aligned}
$$

Remark 1. The solution of the above problems has been pursued with a nonlinear programming local technique, the Sequential Quadratic Programming. Finding the global minimum of these functions, if it exists, can then be a difficult problem. However, a choice that has turned out to be effective in most cases is to set the initial conditions according to the following relations: $\eta_{1}=\eta_{2}=\left\|p_{B}-p_{A}\right\|$.

Finally, it has to be pointed out that a parametrized curve $\mathbf{p}(u, \eta)$ is, by definition, a $G^{3}$ curve and, consequently, a sequence of points interpolated by quintic $G^{3}$ splines results in an overall $G^{3}$ curve. Therefore, it only remains to choose properly those points, but this task will be devolved to the global path planner.

\section{GLOBAL PATH PLANNER}

The method proceeds in two steps: the learning phase and the query phase.

In the first phase, a probabilistic technique will be used to construct a weighted graph whose nodes correspond to collision-free configurations, whose edges correspond to feasible paths between configurations (only path lengths will be stocked).

The environment exploration is done by successively adding a random configuration to the graph, and by trying to connect this configuration to a maximum number of nodes of the graph with the local path planner.

Remark 2. A random configuration is a data set made up of 5 elements: $x_{i}, y_{i}, \theta_{i}, \kappa_{i}, \sigma_{i}$.

The original path planner (Svestka and Overmars, 1995) has been slightly modified in order to achieve a better graph connectivity over complicated access zones. Thus, for a new node $c$, every node belonging to the graph whose distance to the new node is lower than a beforehand fixed distance $d_{\max }$, and whose edges are not zero, is added up. If the resultant value is higher than a heuristic value $n_{\text {max }}$, the new node $c$ is not included in the graph and a new node is generated until it satisfies the previous condition.

This learning phase can be done as an off-line treatment because it only depends on the environment and not on the query configurations.

In the query phase, any given start and goal configuration are connected to the two closest nodes of the graph, using the local planner, and then a graph search between these two nodes is performed. This step is realized with the help of the Dijkstra algorithm (Dijkstra, 1959). As the aim of the planner is to find the shortest possible path, graph weight will be the internodal distance calculated by the local planner.

This planner is, according to the authors, probabilistic complete. This means that any problem which can be solved using an overall path made up of several elementary paths (generated by the local planner) will be solved, provided that the exploration is carried out for a sufficient amount of time.

\section{SIMULATION RESULTS}

First of all, a comparison between different local path planners is shown in Figures 1 and 2 for random start and goal configurations. The shortest path occurs when a low maximal curvature $\left(k_{\max }=0.2\right.$, which could theoretically be covered 


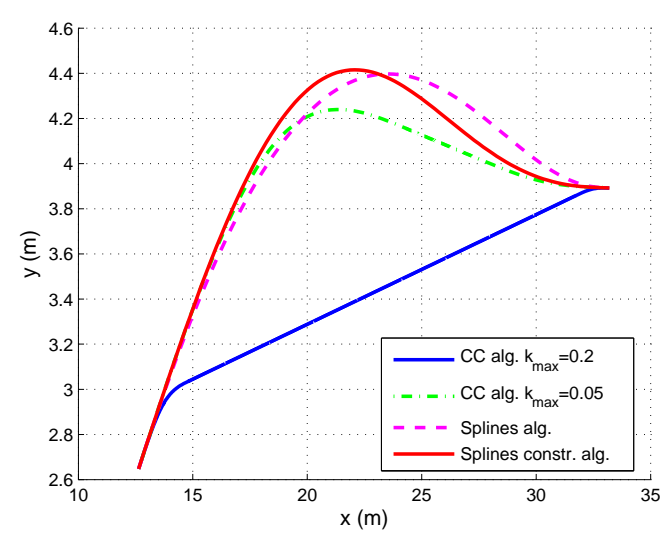

Fig. 1. Path comparison between Continuous Curvature curves with $k_{\max }=0.2$, with $k_{\max }=$ 0.05 , simple Quintic $G^{3}$ splines curves and curvature upper-bounded $\left(k_{\max }=0.03\right)$ quintic $G^{3}$ splines.

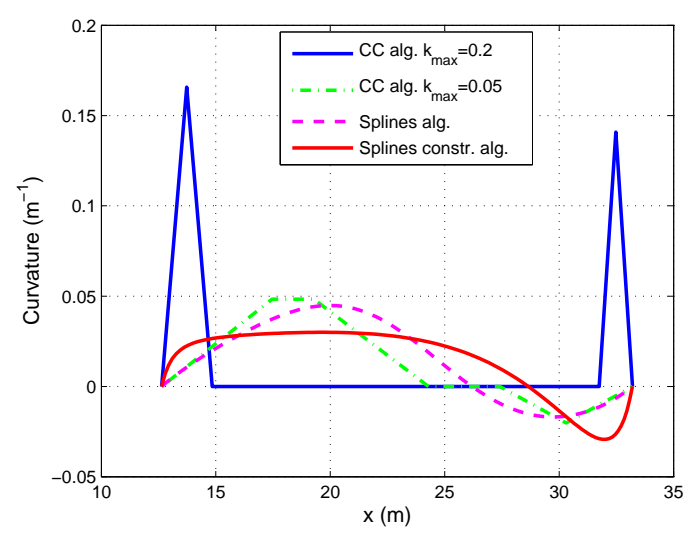

Fig. 2. Curvature profile of previous paths.

by a vehicle running at a speed not higher than $6 \mathrm{~ms}^{-1}$ ) is imposed in Scheuer's CC algorithm. When this constraint becomes more stringent, the path shape looks like those obtained with quintic splines. However, it can be appreciated in Figure 2 a remarkable difference between curvature profiles of those paths. While the CC algorithm produces sheer slope changes in line's segments-clothoids or cercle arcs-clothoids transitions, spline curvature appear to be much softer. Moreover, their curvature have a more versatile structure than CC curves, which are limited to linear pieces. A final remark has to be done concerning the difference between constrained and unconstrained splines curves. The constrained $\left(k_{\max }=0.03\right)$ curvature algorithm succeeds for this particular example, but, in general, it will do it with more difficulty than unconstrained algorithms. Unfortunately, this is the price to pay in order to find more easily trackable paths.

Remark 3. Maximum curvature and curvature derivative values have been obtained for a vehicle running at $v=15 \mathrm{~ms}^{-1}$. From that data, taking into account that a maximal lateral acceleration occurs when tyres saturate $\left(\gamma_{\max } \approx 0.8 g\right)$, and that $\gamma=v^{2} \kappa$, it follows that the maximal curvature value allowed is $\kappa_{\max }=0.03$.

Test results of global path planner for an environment with obstacles are shown in figures 3 and 4. Let $(x=0, y=0.8, \theta=0.02, \kappa=$ $-0.003, \sigma=0.005)$ be the start configuration and $(x=29.93, y=4.51, \theta=0.105, \kappa=-0.03, \sigma=$ 0.004 ) be the goal configuration, the Quintic $G^{3}$ path interpolating those two configurations is the one shown in the figure below. Two families of points can be observed: those who have been randomly drawn (light points) and those to whom another configuration belonging to the graph have managed to be linked (superposed, dark points). As the local path planner have not found a solution to the above specified problem, the global path planner have chosen the most interesting landmark combination for finding the minimal length path. An only intermediate landmark has been found for this particular situation (asterisk).

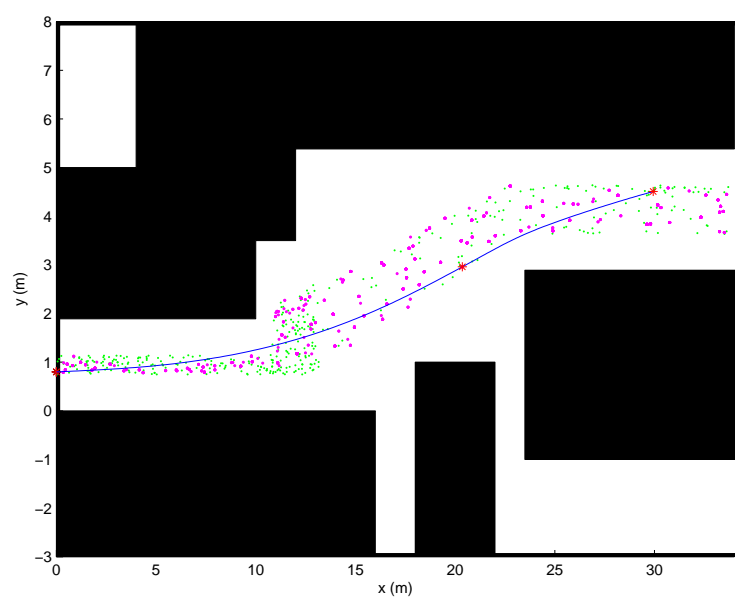

Fig. 3. Path found for random start and goal configuration in an environment with obstacles.

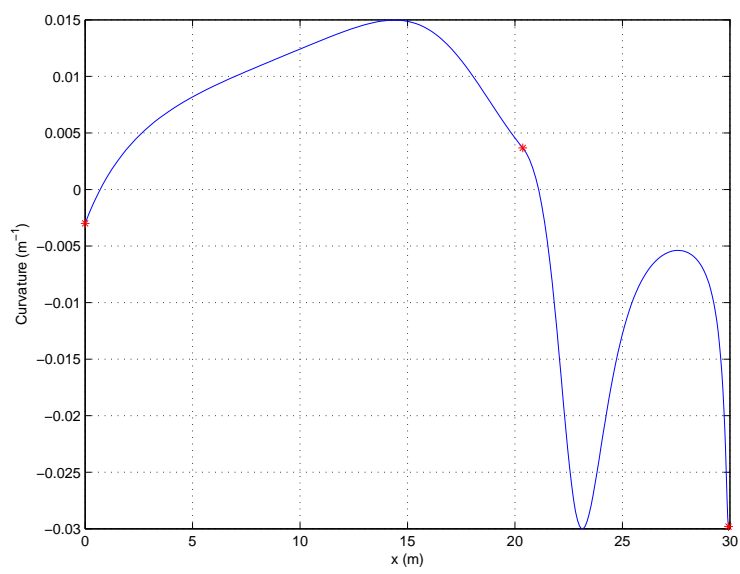

Fig. 4. Curvature profile of previous path. 
Remark 4. Although the optimization problem posed in (10) is closer to provide the shortest path guaranteeing the above curvature constraints, problem formulated as (9) is much more efficient in terms of graph connectivity. This is why the local path planner has been implemented so as to use (9) solution curves.

\section{CONCLUSION}

A new obstacle-avoiding path planning strategy has been presented. The main advantage of this methodology is, thanks to its versatility, its adaptability to non-holonomic vehicles with nearly constant and high velocities. However, a simple modification in local path planner conception could allow to use it also for longitudinal velocity controlled vehicles.

\section{REFERENCES}

Anisi, D. A. (2003). "Optimal Motion Control of a Ground Vehicle". Master's thesis. Royal Institute of Technology (KTH), Stockholm, Sweden.

Berglund, T., H. Jonsson and I. Sderrkust (2003). An obstacle-avoiding minimum variation bspline problem. In: International Conference on Geometric Modelling and graphics. London (UK).

Bianco, C. Guarino Lo and A. Piazzi (2000). Optimal trajectory planning with quintic $g^{2}$ splines. In: Proc. of the IEEE Intelligent Vehicles Symposium. Dearborn, MI (USA). pp. 620-625.

Delingette, H., M. Hbert and K. Ikeuchi (1991). Trajectory generation with with curvature constraint based on energy minimization. In: Proc. of the IEEE-RSJ Int. Conf. on Intelligent Robots and Systems. Vol. 1. Osaka (JP). pp. 206-211.

Dijkstra, E.W. (1959). A note on two problems in connecting with graphs. Numerische Mathmatik 1(5), 269-271.

Dubins, L.E. (1957). On curves of minimal length with a constraint on average curvature, and with prescribed initial and terminal positions and tangents. American Journal of Mathematics (79), 497-516.

Jacobs, P.E. and J. Canny (1989). Planning smooth path for mobile robots. In: Proc. of the IEEE Int. Conf. on Robotics and Automation. Vol. 1. Scottsdale, AZ(US). pp. 2-7.

Kanayama, Y. and B.I. Hartman (1989). Smooth local path planning for autonomous vehicle. In: Proc. of the IEEE-RSJ Int. Conf. on Intelligent Robots and Systems. Vol. 3. Scottsdale, AZ (US). pp. 1265-1270.
Komoriya, K. and K.Tanie (1989). Trajectory design and control of a wheel-type mobile robot using b-spline curve. In: Proc. of the IEEE-RSJ Int. Conf. Robots and Systems. Tsukuba (JP). pp. 398-405.

Laumond, J.P., P.E. Jacobs and M.Taix (1994). A motion planner for non-holonomic mobile robots. Vol. 5. pp. 577-593.

Liscano, R. and D. Green (1989). Design and implementation of a trajectory generator for an indoor mobile robot. In: Proc. of the IEEERSJ Int. Conf. on Intelligent Robots and Systems. Tsukuba (JP). pp. 380-385.

Mirtich, B. (1992). Using skeletons for nonholonomic motion planning. Research report esrc 92-16/ramp 92-6. Department of Electrical Engineering and Computer Science. University of California, Berkeley.

Nelson, W. (1989). Continuous curvature paths for autonomous vehicles. In: Proc. of the IEEE Int. Conf. on Robptics and Automation. Vol. 3. Scottsdale, AZ (US). pp. 12601264.

Piazzi, A., C. Guarino Lo Bianco, M. Bertozzi, A. Fascioli and A. Broggi (2002). Quintic $g^{2}-$ splines for the iterative steering of visionbased autonomous vehicles. IEEE Transactions on Intelligent Transportation Systems 3(2), 27-36.

Piazzi, A., M. Romano and C. Guarino Lo Bianco (2003). $g^{3}$-splines for the path planning of wheeled mobile robots. In: Proc. of the ECC 03 Conference. Cambridge (UK).

Reeds, J.A. and L.A. Shepp (1990). Optimal paths for a car that goes both forward and backwards. Pacific Journal of Mathematics 2(145), 367-393.

Scheuer, A. (1998). Planification de chemins courbure continue pour robot mobile nonholonome. PhD thesis. Inst. Nat. Polytechnique de Grenoble. Grenoble (FR).

Simeon, T., S. Leroy and J.P. Laumond (1997). Computing good holonomic collision-free paths to steer nonholonomic mobile robots. In: Proc. of the IEEE-RSJ Int. Conf. on Intelligent Robots and Systems. Vol. 2. Grenoble (FR). pp. 1004-1009.

Sussmann, H.J. (1997). The markov-dubins problem with angular acceleration control. In: IEEE. Conf. Trnas. Robotics and Automation. San Diego, CA (US).

Svestka, P. and M.H. Overmars (1995). Probabilistic path planning. Technical report. Utrecht University. P.O. Box 80.089, 3508 TB Utrecht, the Netherlands.

Takahashi, A., T. Hongo and Y.Ninomiya (1989). Local path planning and control for agv in positionning. In: Proc. of the IEEE-RSJ Int. Conf. Robots and Systems. Tsukuba (JP). pp. 392-397. 